\title{
Hyperfunctioning Papillary Thyroid Carcinoma with a BRAF Mutation: The First Case Report and a Literature Review
}

\author{
Shinsuke Shinkai ${ }^{a}$ Kenji Ohba a, b Kennichi Kakudo ${ }^{c}$ Takayuki Iwaki $^{d}$ \\ Yoshihiro Mimura ${ }^{\mathrm{e}}$ Akio Matsushita ${ }^{a}$ Go Kuroda ${ }^{a}$ Yuki Sakai $^{\mathrm{a}}$ \\ Nobuhiko Nishino $^{f}$ Kazuo Umemura ${ }^{\text {bd }}$ Takafumi Suda ${ }^{a}$ Shigekazu Sasaki ${ }^{a}$ \\ aSecond Division, Department of Internal Medicine, Hamamatsu University School of Medicine, Shizuoka, Japan; \\ ${ }^{b}$ Medical Education Center, Hamamatsu University School of Medicine, Shizuoka, Japan; 'Department of Pathology \\ and Thyroid Disease Center, Izumi City General Hospital, Osaka, Japan; dDepartment of Pharmacology, Hamamatsu \\ University School of Medicine, Shizuoka, Japan; 'Department of Internal Medicine, American Hospital of Paris, \\ Neuilly sur Seine, France; 'Department of Surgery, Maruyama Hospital, Shizuoka, Japan
}

\section{What Is Known about This Topic?}

- Hyperfunctioning thyroid carcinoma is rare, and its molecular etiology is not clear.

- To the best of our knowledge, there is no reported case of hyperfunctioning thyroid carcinoma with a $B R A F$ mutation (a common somatic mutation in papillary thyroid carcinoma).

\section{What Does This Case Report Add?}

- We identified concurrent BRAF mutation and TSHR polymorphism for the first time in a case of hyperfunctioning malignant thyroid nodule.

- The findings from the present case and our literature search suggest that concomitant activation of oncogenes (in addition to constitutive activation of the thyrotropin receptor-cyclic AMP (cAMP) cascade) plays an important role in the carcinogenesis for hyperfunctioning thyroid nodules.

\section{Keywords}

Autonomous thyroid nodule $\cdot$ BRAF V600E - GNAS.

Hyperfunctioning papillary thyroid carcinoma. TSHR .

Polymorphism

\section{Abstract \\ Introduction: Hyperfunctioning papillary thyroid carcinoma (PTC) is rare and consequently, little information on its mo-}

lecular etiology is available. Although BRAF V600E (BRAF c.1799T >A, p.V600E) is a prominent oncogene in PTC, its mutation has not yet been reported in hyperfunctioning PTC. Case Presentation: Ultrasonography detected a $26-\mathrm{mm}$ nodule in the right lobe of the thyroid gland of a 48-year-old man. Thyroid function tests indicated that he was hyperthyroid with a TSH level of $0.01 \mathrm{mIU} / \mathrm{L}$ (reference range: $0.05-$ 5.00 ) and a free thyroxine level of $23.2 \mathrm{pmol} / \mathrm{L}$ (reference range: 11.6-21.9). TSHR autoantibodies were $<0.8 \mathrm{IU} / \mathrm{L}$ (ref- 
erence value: $<2.0 \mathrm{IU} / \mathrm{L})$. The ${ }^{99 \mathrm{~m}} \mathrm{Tc}$ thyroid scintigram revealed a round, right-sided focus of tracer uptake by the nodule with a decreased uptake in the remainder of the gland. The patient underwent total thyroidectomy because fine-needle aspiration cytology revealed a malignancy. The histopathological diagnosis was conventional PTC. Subsequent mutational analysis of BRAF (exon 15), TSHR (exons 1-10), GNAS (exons 7-10), EZH1 (exon 16), KRAS, NRAS, HRAS (codons 12, 13, and 61), and TERT promoter (C250T and C228T) identified a heterozygous point mutation in BRAF V600E in a tumor tissue sample. In addition, we identified a TSHR D727E polymorphism (TSHR c.2181C>G, p.D727E) in both the tumor and the surrounding normal thyroid tissue.

Discussion and Conclusions: We report a case of hyperfunctioning PTC with a BRAF V600E mutation for the first time. Our literature search yielded 16 cases of hyperfunctioning thyroid carcinoma in which a mutational analysis was conducted. We identified TSHR mutations in 13 of these cases. One case revealed a combination of TSHR and KRAS mutations; the other case revealed a TSHR mutation with a PAX8/ $P P A R G$ rearrangement. These findings suggest that the concomitant activation of oncogenes (in addition to constitutive activation of the TSHR-cyclic AMP cascade) are associated with the malignant phenotype in hyperfunctioning thyroid nodules.

(c) 2021 European Thyroid Association Published by S. Karger AG, Basel

\section{Introduction}

Hyperfunctioning thyroid nodules grow and produce thyroid hormones independently of thyrotropin (TSH) and in the absence of TSH receptor (TSHR) autoantibodies [1]. The classical appearance observed on a thyroid scintigraphy is a circumscribed hyperdense lesion surrounded by hypodense thyroid tissue. Hyperfunctioning thyroid nodules can be classified as benign or malignant, though the incidence of a hyperfunctioning cancerous nodule is extremely low $[1,2]$.

Genomic instability plays an essential role in the progression of thyroid neoplasms, such as papillary thyroid carcinoma (PTC), follicular thyroid adenoma, follicular thyroid carcinoma (FTC), poorly differentiated thyroid carcinoma, and anaplastic thyroid carcinoma [2]. On the basis of clinical, histological, and molecular observations, 3 major pathways are suggested to contribute to the neoplastic proliferation of thyroid follicular cells (PTC, FTC, and hyperfunctioning thyroid nodules). The associated frequent genetic events are BRAF, RAS, and TSHR mutations, respectively. The gain-of-function mutations in
TSHR occur in hyperfunctioning thyroid nodules. However, the genetic alterations in hyperfunctioning thyroid carcinoma remain controversial $[3,4]$. Interestingly, hyperfunctioning thyroid nodules with a BRAF mutation have not yet been reported to the best of our knowledge.

Thus, we describe this case of a hyperfunctioning PTC with a BRAF mutation. Mutational analysis of TSHR revealed D727E polymorphism in both the tumor and surrounding normal thyroid tissue. We also provide a literature review of mutational events leading to a hyperfunctioning thyroid carcinoma.

\section{Case Presentation}

A 48-year-old Japanese man with no family history of benign or malignant thyroid neoplasms, and without exposure to radiation, was found to have a nodule on the right lobe of his thyroid gland from ultrasonography (US) during an annual checkup. Fine-needle aspiration cytology (FNAC) revealed a malignancy, consistent with a PTC. He was $162 \mathrm{~cm}$ tall and $57.8 \mathrm{~kg}$ of weight. His heart rate was 85 beats per min, and his blood pressure was $98 / 66 \mathrm{~mm} \mathrm{Hg}$. Thyroid function tests indicated that he was hyperthyroid with a TSH level of $0.01 \mathrm{mIU} / \mathrm{L}$ (reference range: $0.05-$ 5.00 ), a free thyroxine level of $23.2 \mathrm{pmol} / \mathrm{L}$ (reference range: $11.6-$ 21.9 ), and a free tri-iodothyronine level of $6.6 \mathrm{pmol} / \mathrm{L}$ (reference range: 3.5-6.2) as measured by ECLusys (Roche Diagnostics K.K., Tokyo, Japan). Serum thyroglobulin (Tg), anti-Tg antibodies, and TSHR autoantibody levels were $62.1 \mu \mathrm{g} / \mathrm{L}$ (reference range, <33.7), $<10 \mathrm{IU} / \mathrm{L}$ (reference range, $<28$ ), and $<0.8 \mathrm{IU} / \mathrm{L}$ (reference range, $<2.0 \mathrm{IU} / \mathrm{L}$ ) as measured by ECLusys, respectively. B-mode US revealed a moderately hypoechoic solid nodule with the largest diameter of $26 \mathrm{~mm}$ (Fig. 1a). The nodular lesion had a smooth margin and no risk features, with a European Thyroid Imaging and Reporting Data System (EU-TIRADS) score of 4 (intermediate risk). The nodular lesion showed hypervascularity on color Doppler US (Fig. 1b) and low elasticity on elastography. The ${ }^{99 \mathrm{~m}} \mathrm{Tc}$ thyroid scintigram revealed a round, right-sided hyperdense lesion by the nodule concomitant with a decreased uptake in the remainder of the gland (Fig. 1c). These findings were consistent with the profile for a hyperfunctioning thyroid nodule. The patient underwent total thyroidectomy, because multiple lateral lymph node metastases were suspected by the preoperative US (cN1b). The histopathological diagnosis of the patient was a conventional PTC with tall cell features (pT2, pEx0, pN1b, and M0) (Fig. 1d).

To conduct mutational analysis, we extracted DNA from stored formalin-fixed, paraffin-embedded tissues. We performed PCR amplification and Sanger sequencing to analyze the TSHR (exons 1-10), GNAS (exons 7-10), EZH1 (exon 16), BRAF (exon 15), KRAS, NRAS, HRAS (codons 12, 13, and 61), and the TERT promoter (C250T and C228T). We also performed cancer panel analysis to analyze the TSHR, BRAF, KRAS, NRAS, and HRAS mutations. Analytic sensitivity of this method was approximately $0.1 \%$ of mutant alleles. Details of the method are described in online suppl. information (see www.karger.com/doi/10.1159/000513552 for all online suppl. material). 

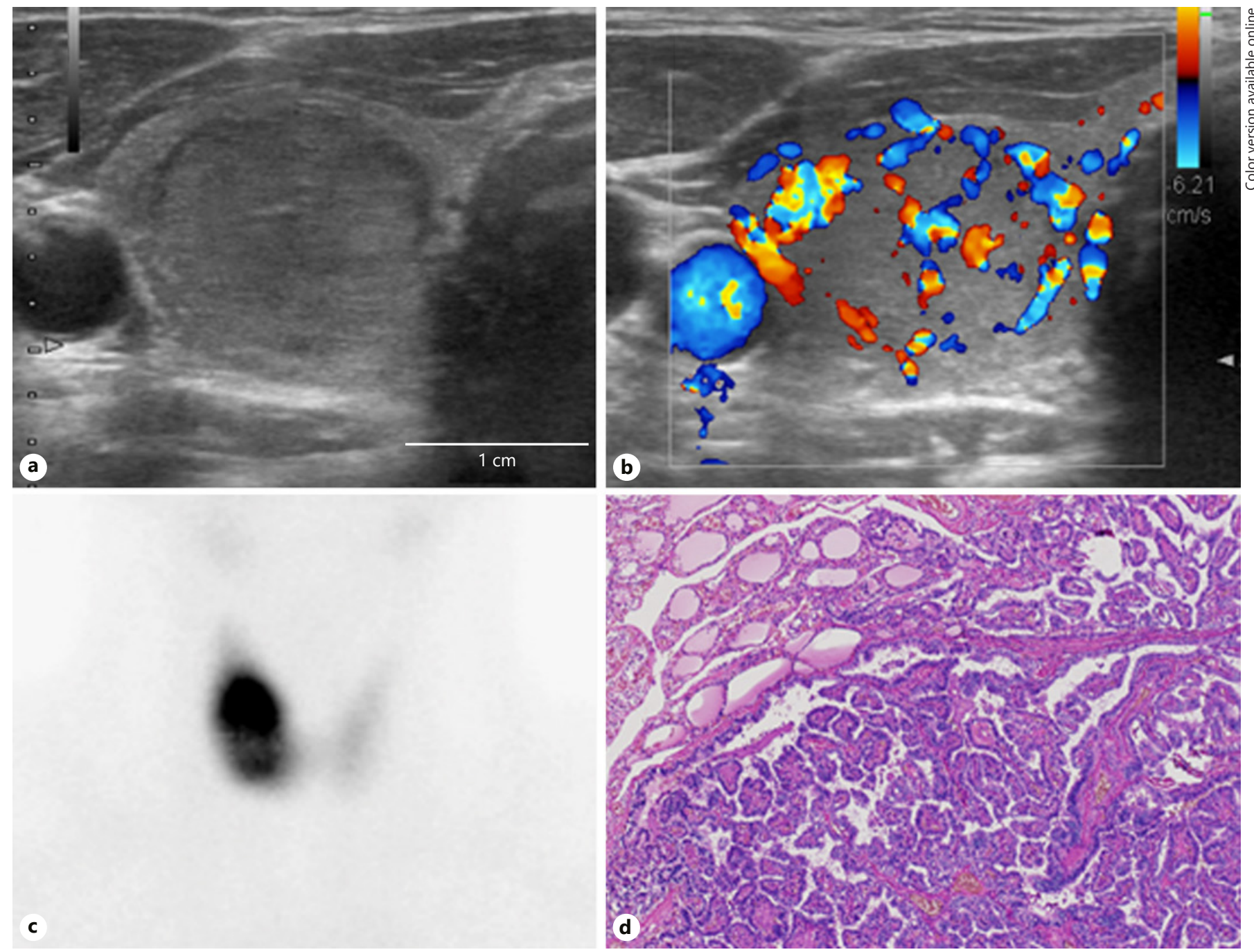

Fig. 1. Hyperfunctioning PTC with concurrent $B R A F$ V600E mutation (BRAF c.1799T>A, p.V600E) and TSHR polymorphism (TSHR c.2181C > G, p.D727E) in a 48-year-old man. a Longitudinal view of B-mode US revealing a moderately hypoechoic nodule with a smooth margin and the largest diameter of $26 \mathrm{~mm}$. b Color Doppler US showing peripheral and intranodular hypervascular-

ity. ${ }^{99 \mathrm{~m}} \mathrm{Tc}$ thyroid scintigraphy revealing a round, right-sided focus of tracer uptake by the nodule with a decreased uptake in the remainder of the gland. $\mathbf{d}$ Resected thyroid nodule demonstrating invasive growth into thyroid parenchyma. Note the papillary growth pattern of classic type PTC (hematoxylin-eosin stain, $\times 20$ ). PTC, papillary thyroid carcinoma; US, ultrasonography.

The result revealed a heterozygous $B R A F$ V600E mutation (BRAF c.1799T $>$ A, p.V600E) in the tumor (Fig. 2a), with wild-type $B R A F$ in the surrounding thyroid tissue (Fig. $2 \mathrm{~b}$ ). In addition, we identified heterozygous point mutations of TSHR in exon 10 (GAC to GAG) resulting in the substitution of aspartic acid for glutamic acid at codon 2,181 (TSHR c.2181C>G, p.D727E) in both the tumor and the surrounding normal thyroid tissue (Fig. 2c). The allelic frequencies of BRAF V600E and TSHR D727E in the tumor tissue were 69.6 and 51.2\%, respectively. Subsequent immunohistochemical analyses demonstrated that staining with BRAF (VE1) led to a positive result in the cytoplasm of tumor cells but not the

surrounding normal thyroid tissue cells (Fig. 2d). In contrast, the tumor and the surrounding thyroid tissue were all positive for $\mathrm{Tg}$ (Fig. 2e, f).

We classified the patient as intermediate-risk group and decided to conduct adjuvant therapies according to the 2018 edition of Japanese Clinical Guidelines for Treatment of Thyroid Tumor [5]. First, we initiated TSH-suppressive therapy with levothyroxine after the surgery. Second, he received radioactive iodine ablation. One year after the initial treatment, the patient displayed a serum $\mathrm{Tg}$ level of $4.0 \mu \mathrm{g} / \mathrm{L}$ and a radioactive iodine whole body scan was negative for disease. 

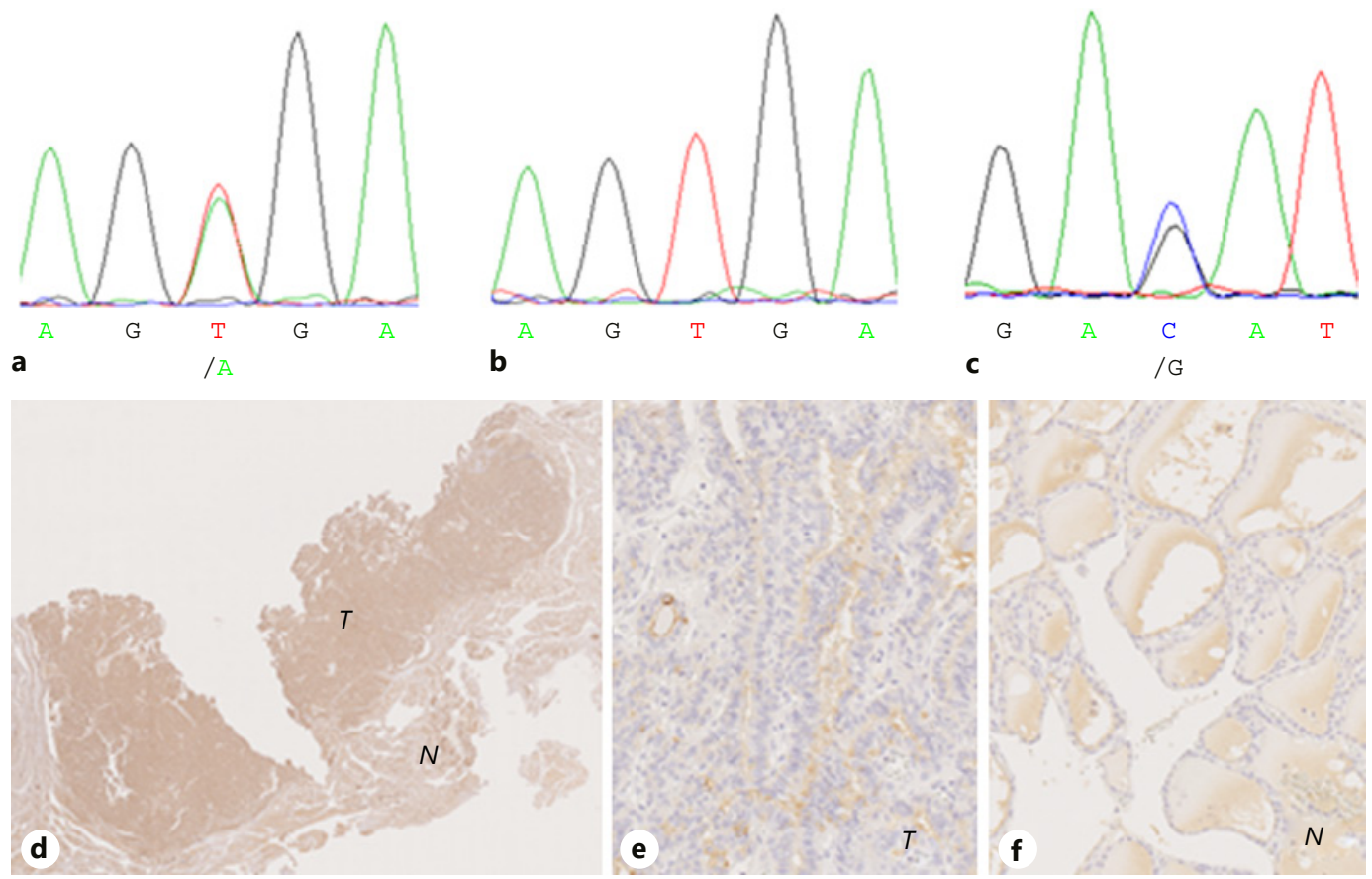

Fig. 2. Mutational and immunohistochemical analyses. a Molecular analysis of genomic DNA extracted from the tumor tissue revealing a heterozygous point mutation of $B R A F$ in exon 15 (GTG to $G A G)$ resulting in the substitution of valine for glutamic acid at codon 600 (BRAF c.1799T >A, p.V600E). b Only the wild-type $B R A F$ sequence is present in the surrounding normal thyroid tissue. c Heterozygous point mutations of TSHR in exon 10 (GAC to GAG) resulting in the substitution of aspartic acid for glutamic

\section{Discussion/Conclusion}

Here, we report an unusual case of hyperfunctioning PTC with concurrent BRAF V600E mutation and TSHR D727E polymorphism. Due to scarcity of information about genetic alterations in hyperfunctioning thyroid carcinoma, we searched PubMed for cases of hyperfunctioning thyroid carcinoma. We excluded reports that did not describe the thyroid function tests and the hot nodule on thyroid scintigraphy. Eventually, our literature search yielded 16 cases of hyperfunctioning thyroid carcinoma wherein a mutational analysis was conducted (online suppl. information). TSHR mutations were identified in 13 of these cases; notably one revealed a mutation in both TSHR and KRAS [6], and another revealed a mutation in TSHR with a rearrangement in PAX8/PPARG [7].

To the best of our knowledge, this is the first documented case of a hyperfunctioning thyroid carcinoma acid at codon 2,181 (TSHR c.2181C >G, p.D727E) are present in both the tumor tissue and the surrounding normal thyroid tissue. d Immunohistochemical staining with BRAF (VE1) led to a positive result in the cytoplasm of the tumor tissue $(T)$ cells but not the surrounding normal thyroid tissue $(N)$ cells $(\times 10)$. e, f Immunohistochemical staining with thyroglobulin led to positive results in the cytoplasm of both the tumor tissue and the surrounding thyroid tissue cells $(\times 40)$. with a $B R A F$ mutation. The $B R A F \mathrm{~V} 600 \mathrm{E}$ mutation is the most common type of BRAF mutations in PTC and is typically found in conventional PTC and tall cell variants of PTC [3]. Considering the reported frequency of $B R A F$ mutations (approximately $30-80 \%$ in sporadic adult PTCs [3]), it is surprising that no cases of hyperfunctioning thyroid carcinoma with a $B R A F$ mutation have been reported yet. This may be because $B R A F \mathrm{~V} 600 \mathrm{E}$ is associated with the loss of expression of iodine metabolism genes in thyroid tissue, such as TSHR [8].

Regarding the etiology of a hyperfunctioning thyroid nodule, a somatic point mutation in GNAS, which codes for the a subunit of the stimulatory G protein $(\mathrm{Gs} \alpha)$, was first reported by Lyons et al. [9] in hyperfunctioning follicular adenoma. In contrast, Parma et al. [10] identified constitutively activating somatic TSHR mutations as the cause of most hyperfunctioning nodules. In addition, Calebiro et al. [11] recently identified EZH1 mutations as 
the second most frequent genetic alteration in hyperfunctioning thyroid adenomas. TSH-bounded TSHR (coupled to the Gsa), stimulates adenylate cyclase and generates its secondary messenger (cAMP), thereby promoting growth of thyroid cells [1]. Therefore, other mutational or epigenetic events within the TSHR-Gsa-cAMP signaling cascade are likely to occur in TSHR, GNAS, and EZH1 mutation-negative nodules $[1,11]$.

There are postulates that constitutive activation of the cAMP cascade alone is insufficient to induce malignancy in thyroid follicular cells $[2,3]$ for the following reasons. First, a gain-of-function TSHR mutation is reported in only a few cases of carcinoma, although alterations in $T S H R$ are frequently observed in benign hyperfunctioning thyroid nodules [12]. Second, the incidence of thyroid cancer is low in patients with McCune-Albright syndrome, which results from germline GNAS activation [13]. Third, oncogenic mutations were previously reported in hyperfunctioning thyroid carcinoma. For example, Niepomniszcze et al. [6] described a case of hyperfunctioning FTC in which a mutational analysis revealed somatic mutations in both TSHR and KRAS. Moreover, Lado-Abeal et al. [7] reported a hyperfunctioning FTC harboring a somatic TSHR mutation in a patient with a $P A X 8 / P P A R G$ rearrangement mosaicism. Here we identified a BRAF V600E mutation, which contributed to the induced malignancy. Whether the TSHR D727E variant may also have contributed is not clear, as approximately $10 \%$ of healthy individuals possess this polymorphism [14]. We recommend further studies to clarify whether concurrent BRAF V600E mutation and TSHR D727E polymorphism may predispose patients to develop hyperfunctioning carcinoma as part of a multi-hit process.

From the physician's point of view, one may wonder why FNAC was conducted before determining serum TSH level in our case. The American Thyroid Association management guidelines for adult patients with thyroid nodules recommended that no cytologic evaluation is necessary if serum TSH is subnormal [15]. In contrast, the Japanese Thyroid Association management guidelines for this disorder recommended that FNAC is mandatory based on US findings but not serum TSH levels [16]. This discrepancy may be due to the higher incidence of malignancy in hyperfunctioning thyroid nodule (5.1-11.8\% in different series) reported in the Japanese population [16] compared to Western populations [1]. This is because different etiologies in iodine-deficient Europe and iodine-sufficient Japan may result in the different incidences of malignancy in hyperfunctiong thyroid nodules. Although the incidence of hyperfunctioning carcinoma is low in Western populations, FNAC may play an important role in the diagnosis of clinical thyrotoxicosis.

The limitations of the present study include a small sample size (only one patient), and frozen tissue was not obtained during the thyroidectomy. Since it has been reported that DNA extracted from stored formalin-fixed, paraffin-embedded tissue is highly fragmented, we may have missed some genetic alterations.

In conclusion, we identified a BRAF mutation for the first time in the case of hyperfunctioning thyroid carcinoma. We hypothesize that concomitant activation of oncogenes (in addition to constitutive activation of the TSHR-cAMP cascade) played an important role in the carcinogenesis for hyperfunctioning thyroid nodules.

\section{Acknowledgements}

The authors would like to acknowledge the technical support from Dr. Tomohiro Arakawa and Mrs. Hiroko Nakamura. This work was supported by HUSM Grant-in-Aid. Portions of this manuscript were presented at the Endocrine Society's Annual Meeting held in San Francisco, 2020 (Conference canceled).

\section{Statement of Ethics}

The Institutional Review Board of the Hamamatsu University School of Medicine approved this study (19-174), which was congruent with the Declaration of Helsinki. We obtained written informed consent from the patient for publication of this case report and any accompanying images.

\section{Conflict of Interest Statement}

The authors have no conflicts of interest to declare.

\section{Funding Sources}

No specific funding was obtained for the present study.

\section{Author Contributions}

S. Shinkai drafted the original manuscript. K.O. is the corresponding author and organized the study. K.K., T.I., K.U., T.S., and S. Sasaki were involved in reviewing and editing the manuscript. Y.M., A.M., G.K., Y.S., and N.N. contributed to the acquisition of data. 


\section{References}

1 Paschke R. Toxic adenoma and toxic multinodular goiter. In: Braverman LE, Cooper DS, editors. Werner's and Ingbar: the thyroid a fundamental and clinical text. 10th ed. Philadelphia: Lippincott Williams \& Wilkins; 2013. p. 400-9.

2 Kondo T, Ezzat S, Asa SL. Pathogenetic mechanisms in thyroid follicular-cell neoplasia. Nat Rev Cancer. 2006;6(4):292-306.

3 Sobrinho-Simoes M, Maximo V, Rocha AS, Trovisco V, Castro P, Preto A, et al. Intragenic mutations in thyroid cancer. Endocrinol Metab Clin North Am. 2008;37(2):333-62.

4 Jaeschke H, Undeutsch H, Patyra K, Löf C, Eszlinger M, Khalil M, et al. Hyperthyroidism and papillary thyroid carcinoma in thyrotropin receptor $\mathrm{D} 633 \mathrm{H}$ mutant mice. Thyroid. 2018;28(10):1372-86.

5 The Japan Association of Endocrine Surgeons and the Japanese Society of Thyroid Surgery. 2018 Japanese clinical guidelines for treatment of thyroid tumor. Official J Jpn Asso Endocr Surg Jpn Soc Thyroid Surg. 2018;35(Suppl 3):1-88. [in Japanese].

6 Niepomniszcze H, Suárez H, Pitoia F, Pignatta A, Danilowicz K, Manavela M, et al. Follicular carcinoma presenting as autonomous functioning thyroid nodule and containing an activating mutation of the $\mathrm{TSH}$ receptor
(T620I) and a mutation of the Ki-RAS (G12C) genes. Thyroid. 2006;16(5):497-503.

7 Lado-Abeal J, Celestino R, Bravo SB, GarciaRendueles ME, de la Calzada J, Castro I, et al. Identification of a paired box gene 8-peroxisome proliferator-activated receptor gamma (PAX8-PPARgamma) rearrangement mosaicism in a patient with an autonomous functioning follicular thyroid carcinoma bearing an activating mutation in the TSH receptor. Endocr Relat Cancer. 2010;17(3):599-610.

8 Liu D, Hu S, Hou P, Jiang D, Condouris S, Xing M. Suppression of BRAF/MEK/MAP kinase pathway restores expression of iodidemetabolizing genes in thyroid cells expressing the V600E BRAF mutant. Clin Cancer Res. 2007;13(4):1341-9.

9 Lyons J, Landis CA, Harsh G, Vallar L, Grünewald K, Feichtinger H, et al. Two G protein oncogenes in human endocrine tumors. Science. 1990;249(4969):655-9.

10 Parma J, Duprez L, Van Sande J, Cochaux P, Gervy C, Mockel J, et al. Somatic mutations in the thyrotropin receptor gene cause hyperfunctioning thyroid adenomas. Nature. 1993; 365(6447):649-51.

11 Calebiro D, Grassi ES, Eszlinger M, Ronchi CL, Godbole A, Bathon K, et al. Recurrent EZH1 mutations are a second hit in autono- mous thyroid adenomas. J Clin Invest. 2016; 126(9):3383-8.

12 Stephenson A, Lau L, Eszlinger M, Paschke R. The thyrotropin receptor mutation database update. Thyroid. 2020;30(6):931-5.

13 Collins MT, Sarlis NJ, Merino MJ, Monroe J, Crawford SE, Krakoff JA, et al. Thyroid carcinoma in the McCune-Albright syndrome: contributory role of activating Gs alpha mutations. J Clin Endocrinol Metab. 2003;88(9): 4413-7.

14 Gabriel EM, Bergert ER, Grant CS, van Heerden JA, Thompson GB, Morris JC. Germline polymorphism of codon 727 of human thyroid-stimulating hormone receptor is associated with toxic multinodular goiter. J Clin Endocrinol Metab. 1999;84(9):3328-35.

15 Haugen BR, Alexander EK, Bible KC, Doherty GM, Mandel SI, Nikiforov YE, et al. 2015 American thyroid association management guidelines for adult patients with thyroid nodules and differentiated thyroid cancer: the American thyroid association guidelines task force on thyroid nodules and differentiated thyroid cancer. Thyroid. 2016;26(1):1-133.

16 The Japan Thyroid Association. 2013 Japanese guideline for management of thyroid nodules. Tokyo: Nankodo; 2013. [in Japanese]. 